\title{
Producción de forraje hidropónico de maíz (Zea mays L.) utilizando fertilizantes químicos y orgánicos
}

\section{Hydroponic maize (Zea mays $L_{\text {. }}$ ) forage production using chemical and organic fertilizers}

Valverde Yhony Alfredo $^{1 *}$, Mera Aurora ${ }^{2}$, Castro Carlos ${ }^{3}$, Gabriel-Ortega Julio ${ }^{4 *}$

\begin{tabular}{l} 
Datos del Artículo \\
\hline Gestión de Proyecto Socio Productivos. \\
Facultad de Ciencias Naturales y de la \\
Agricultura, km 1.5 vía a Noboa, Campus \\
Los Ángeles de la Universidad Estatal del \\
Sur de Manabí UNESUM, Jipijapa \\
Manabí. Tel. (593) 052600229. \\
2 Facultad de Ciencias Naturales y de la \\
Agricultura. Ingeniería Agropecuaria km \\
1.5 vía a Noboa, Campus Los Ángeles de \\
la Universidad Estatal del Sur de Manabí \\
UNESUM, Jipijapa Manabí. Tel. (593) \\
052600229. \\
3 Administración Ambiental. Facultad de \\
Ciencias Naturales y de la Agricultura, km \\
1.5 vía a Noboa, Campus Los Ángeles de \\
la Universidad Estatal del Sur de Manabí \\
UNESUM, Jipijapa Manabí. Tel. (593) \\
052600229. \\
4 Producción Agraria y Aplicaciones \\
Biotecnológicas. PROMETEO/ATENEO \\
de la Facultad de Ciencias Naturales y de \\
la Agricultura, km 1.5 vía a Noboa, \\
Campus Los Ángeles de la Universidad \\
Estatal del Sur de Manabí UNESUM, \\
Jipijapa Manabí. Tel. (593) 052600229. \\
*Dirección de contacto: \\
Julio Gabriel-Ortega \\
Yhony A Valverde \\
E-mail: jgabriel@proinpa.org \\
$\quad$ alfredojvl10@ @hotmail.com \\
Palabras clave: \\
Hidropónico, \\
producción, \\
fertilizantes, \\
inorgánico, \\
orgánico, \\
forraje. \\
\hline
\end{tabular}

J Selva Andina Biosph. 2017; 5(2):144-151.

\section{Historial del artículo.}

Recibido abril, 2017. Devuelto agosto 2017

Aceptado septiembre, 2017

Disponible en línea, noviembre 2017.

Editado por:

Selva Andina

Research Society

\section{Resumen}

La provincia de Manabí ocupa el primer lugar en la producción cárnica en Ecuador, la deficiencia de alimento durante la época seca es uno de sus principales problemas, se plantea la producción de forraje verde hidropónico (FVH) como alternativa de solución, para lo que se plantearon los siguientes objetivos: $i$ ) determinar el fertilizante apropiado para la producción hidropónica de maíz, ii) establecer la variedad de maíz con mayor rendimiento como FVH y iii) definir el tiempo de oreo ideal en la explotación de FVH. Para desarrollar la investigación se aplicó $2 \mathrm{~mL} / \mathrm{L}$ de agua de un fertilizante inorgánico (FI) a base de fosforo y se aplicó $2 \mathrm{~g} / \mathrm{L}$ de agua de micorriza en polvo, se contrastaron en las variedades de maíz blanco y amarillo, con oreos de 48 y 72 h respectivamente. El diseño experimental aplicado fue completamente al azar con arreglo factorial de $2 \times 2 \times 2$ con ocho tratamientos, generando 64 unidades experimentales, considerando como variables de respuesta, peso, tamaño de raíz y altura de hojas del FVH. Los resultados determinaron mayor peso del maíz blanco con FI con $1.60 \mathrm{~kg}$ en bandejas de 40 x $50 \mathrm{~cm}$ y con $0.45 \mathrm{~kg}$ de maíz por bandeja, a nivel de altura no se determinó diferencia estadística y en lo que respecta al tamaño de raíz, el mejor tratamiento fue el maíz blanco con FI con 12.49 $\mathrm{cm}$ de longitud. Se concluye que el maíz blanco responde de mejor manera a la FI en condiciones hidropónicas, con un tiempo de oreo de $48 \mathrm{~h}$.

(C) 2017. Journal of the Selva Andina Biosph. Bolivia. Todos los derechos reservados.

Abstract

The province of Manabí occupies the first place in the meat production in Ecuador, the deficiency of food during the dry season is one of its main problems. The production of green hydroponic fodder (GHF) is proposed as an alternative solution, for which the following objectives were: i) determine the appropriate fertilizer for the hydroponic production of maize, ii) to establish the maize variety with the highest yield as GHF and iii) to define the appropriate harvest time in the exploitation of GHF. In order to develop this research, $2 \mathrm{~mL} / \mathrm{L}$ of water of an inorganic fertilizer (IF) based on phosphorus was applied and $2 \mathrm{~g} / \mathrm{L}$ of powdered mycorrhizal water were applied, which were tested in the varieties of white and yellow maize, With airing of 48 and $72 \mathrm{~h}$ respectively. The experimental design was completely randomized with factorial arrangement of $2 \times 2 \times 2$ with eight treatments, generating 64 experimental units, considering as variables of response, weight, root size and leaf height of the GHF. The results determined higher weight of white maize with FI with $1.69 \mathrm{~kg}$ in trays of $40 \times 50 \mathrm{~cm}$ and with $0.45 \mathrm{~kg}$ of maize per tray, at height level no statistical difference was determined and for root size, the best treatment was white corn with FI with $12.49 \mathrm{~cm}$ in length. It is concluded that white maize 


\begin{tabular}{l||c}
\hline Key words: & responds better to IF under hydroponic conditions, with an airing time of $48 \mathrm{~h}$. \\
$\begin{array}{l}\text { Hydroponic, } \\
\text { production, } \\
\text { fertilizer, } \\
\text { inorganic, } \\
\text { organic, } \\
\text { forage. }\end{array}$ & ○ 2017. Journal of the Selva Andina Biosph. Bolivia. All rights reserved. \\
\hline
\end{tabular}

\section{Introducción}

La provincia de Manabí tiene por su ubicación geográfica muchas bondades naturales, entre las que se destacan: montañas, ríos, lagos, cascadas, humedales, bosques seco tropical y húmedo, $350 \mathrm{Km}$ de playas, islas, cuevas, valles, colinas, etc. (Ecuale 2016), con precipitaciones promedio de 250 a $300 \mathrm{~mm}$, que la hace susceptible a problemas propios de la sequía, como escases de alimento para ganado bovino, cerdos, caballo u otros animales de interés productivo. Situación que ocurre de manera particular en el bosque seco, que comprende particularmente la franja costera, la misma cubre un $50 \%$ del territorio sur manabita.

Los problemas que se han producido en el sector pecuario en los últimos años en la provincia de Manabí, han generado cuantiosas pérdidas económicas por bajas en el rendimiento productivo y en el peor de los casos por incremento de las tasas de mortalidad. Tal es problema que en muchas ocasiones ha provocado que se decreten estados de emergencia, procurando salvar de la muerte al ganado vacuno, citamos como ejemplo lo acontecido en el 2015, donde el Comité de Operaciones Emergentes (COE) parroquial solicitar ayuda para más de 2000 habitantes de Julcuy y para rescatar a unas 600 vacas, pues 3 meses antes se habían muerto unas 30 cabezas de ganado (Eluniverso 2015).

La provincia de Manabí cuenta con una producción ganadera de al menos un millón de cabezas, liderando la producción de carne a nivel nacional y ocupando un tercer lugar en la producción láctea, bondades que exigen por otro lado una alta producción de pastos y forrajes, que en la época seca escasean (mayo a diciembre), lo que representa un reto para los investigadores agropecuarios y por tanto una invitación a tomar medidas que permitan superar la problemática alimenticia en esta época del año.

145
En este sentido la Universidad Estatal del Sur de Manabí "UNESUM" ha iniciado un programa de investigación de producción de forraje verde hidropónico $(\mathrm{FVH})$ de maíz, a fin de contribuir a la solución de un problema que año a año aqueja a los productores de ganado vacuno y caprino de manera particular.

El forraje hidropónico es una alternativa válida en sectores que sufren de escases de agua para riego, o que por las condiciones propias del trópico, resulta difícil durante la época seca la producción en el suelo. El FVH es una tecnología de producción de biomasa vegetal obtenida a partir del crecimiento inicial de las plantas en los estados de germinación y crecimiento temprano de plántulas a partir de semillas viables. El FVH o "green fodder hydroponics" en un pienso o forraje vivo, de alta digestibilidad, calidad nutricional y muy apto para la alimentación animal (FAO 2001, Rivera et al. 2010).

Otro aspecto a destacar del FVH además de la calidad, es la producción pues de $1 \mathrm{q}$ de semilla, en apenas 10 días se puede convertir en $4 \mathrm{q}$ de alimento fresco y sin mayores costos, pues no se requiere de sofisticadas tecnologías para su producción, se puede producir en cualquier época del año, y dado el bajo consumo de agua es factible para sectores propios del bosque seco.

La zona sur de la provincia de Manabí a diferencia del sector norte, cuenta con pequeños ganaderos que poseen entre 10 a 20 cabezas de ganado que materializan su producción en el bosque seco, por tanto sufren escases de pastos y forrajes como alimento para sus animales. El FVH resulta una alternativa viable, si consideramos que este tipo de producción ahorra 10 veces la cantidad de agua que se utilizaría en una producción en campo abierto.

Consecuentemente, la búsqueda de metodologías alternativas de producción de forraje en las cuales se considere 
el ahorro de agua, altos rendimientos por $\mathrm{m}^{2}$ ocupado, calidad nutricional, flexibilidad en la transferencia y mínimos impactos negativos sobre el medio ambiente es de particular importancia (López et al. 2009, Villavicencio 2014).

Otro criterio comúnmente utilizado para determinar la calidad del forraje es la digestibilidad. El contenido de fibra detergente ácida (FDA) es una cuantificación de la fracción indigerible. En el FVH la FDA varía con el tiempo de cosecha: se observan valores menores en la etapa inicial y valores mayores en la etapa final. El requerimiento de fibra por el ganado es un factor importante en diversos procesos fisiológicos. La FDA es el mejor indicador de los requerimientos de fibra para una fermentación saludable en el rumen. (Maldonado-Torres et al. 2013).

Los daños ambientales que se contra restan con la explotación sustentable de FVH son: compactación y acides de los suelos por abuso y mal manejo de los suelos, explotación irracional del agua, se evita agotamiento de los pozos por abuso de su capacidad hídrica, daños al ecosistema local entre los más destacados. Definiendo por tanto que la explotación de FVH contribuye al cuidado del medio ambiente, lo cual es altamente significativo si consideramos que los sectores secos o áridos son susceptibles a afectaciones ambientales.

Sin embargo es oportuno citar varias ventajas, que implica la producción de FVH: Ahorro de agua: 1 a 2 L para producir entre 2 a $3 \mathrm{~kg}$ de FVH. $8 \mathrm{~L}$ para $1 \mathrm{~kg}$ de materia seca. Menor costo de producción y eficiencia en el uso de espacio: En general, el costo de producción de FVH es 10 veces menor comparado con la producción de cualquier forraje en espacios abiertos. Eficiencia en el tiempo de producción: La producción de FVH tiene un ciclo de 12 a 20 días, dependiendo de las condiciones de temperatura, luminosidad, humedad, entre otros. Calidad del forraje: Cosechado a los 12 días y con unos 20 a $30 \mathrm{~cm}$ de altura, el FVH es rico en vitaminas A y E, contiene carotenoides, además, importantes cantidades de hierro, calcio, fósforo, su digestibilidad es alta debido a baja presencia de lignina y celulosa. Inocuidad: Con el FVH los animales no comen malezas, pastos pisoteados o con desechos orgánicos de los mismos animales, de esta forma los procesos de metabolismo y absorción del alimento es óptima (Abarca-Reyes et al. 2014, Villavicencio 2014). Los beneficiarios del proceso investigativo sobre producción de FVH, serán los productores ganaderos de bovinos y caprinos, que son explotados preferencialmente en el bosque seco, por lo que es oportuno a futuro incluirlos en el desarrollo productivo, a fin de generar cambios culturales en la producción y alimentación del ganado bovino de manera particular. Por lo mencionado se plantearon los siguientes objetivos: $i$ ) determinar el fertilizante apropiado para la producción hidropónica de maíz, ii) establecer la variedad de maíz con mayor rendimiento como FVH y iii) definir el tiempo de oreo ideal en la explotación de FVH.

\section{Materiales y métodos}

Sitio de investigación. La investigación se realizó en la Finca experimental de la Universidad Estatal del Sur de Manabí "UNESUM" ubicada en el cantón Jipijapa, km 5 de la vía Jipijapa-Noboa, situado geográficamente al sur de la provincia de Manabí.

Implementación del experimento. Para la realización del ensayo, se construyó un invernadero de sarán de 7 x $7 \mathrm{~m}$ con estructura de caña guadua, se construyeron lechos de madera para sostén de las bandejas, estos lechos fueron de $1.20 \mathrm{~cm}$ de alto, lo que permitió que se construyeran tres pisos de producción de FVH de maíz, las bandejas que se emplearon eran de 40 x $50 \mathrm{~cm}$, con $10 \mathrm{~cm}$ de alto, hechas completamente de madera y recubiertas de plástico, a las que se le hacían agujeros para el drenaje del agua, cada bandeja contaba con un equivalente a 0.2 $\mathrm{m}^{2}$, sembrando $0.45 \mathrm{~kg}$ de semillas en cada una de las bandejas.

El experimento fue implementado en un invernadero de $49 \mathrm{~m}^{2}$, aplicando un diseño experimental completamente aleatorio con arreglo factorial $2 \times 2 \times 2$, con ocho tratamientos, dando lugar a que se 
generen 64 unidades experimentales (UE), los factores de estudio fueron: Factor A. Variedades de maíz (A1=Maíz amarillo variedad 542 y A2. Maíz blanco variedad 528), el Factor B tiempos de oreo (B1=48 h y B2=72 h), y el Factor C. Tipos de Enraizadores, se emplearon $\mathrm{C} 1$. Micorriza solida de la empresa Huxtable (Compuesta de arcilla expandida de color café ocre en mezcla con perlita, con una densidad: $0.675 \mathrm{~g} / \mathrm{L}$. Porcentaje de humedad: máximo 1520\%. Grado de Infección: 50 unidades) y C2. Raízfares, un producto a base de fosforo y nitrógeno de la empresa Edifarm (compuesto por $9 \%$ de nitrógeno total $(\mathrm{N}), 42.5 \%$ de anhídrido fosfórico $\left(\mathrm{P}_{2} \mathrm{O}_{5}\right), 11 \%$ de potasio $\left(\mathrm{K}_{2} \mathrm{O}\right), 12 \%$ de aminoácidos, $18 \%$ de bioactivadores biológicos y $7.5 \%$ de tensoactivos orgánicos).

Con respecto a los enraizadores, se empleó como fertilizante orgánico la micorriza con una dosis de 2 $\mathrm{g} / \mathrm{L}$ de agua, y raízfares como fertilizante inorgánico, con una dosis de $2 \mathrm{~mL} / \mathrm{L}$ de agua.

Se garantizó la aleatoriedad para la ubicación de los tratamientos en cada una de las unidades experimentales, dentro del área que fue completamente homogénea. La temperatura promedio dentro del invernadero fue de $25^{\circ} \mathrm{C}$.

Manejo del experimento. Se lavó y desinfectó las semillas de maíz con cloro al $1 \%$, luego se procedió a seleccionar las semillas [que no estuvieran partidas, o afectadas por el gorgojo (Sitophilus zeamais)], una vez seleccionado se lo unificó en diferentes recientes para dejarlos al remojo por $24 \mathrm{~h}$. Transcurrido este tiempo, se sacó del remojo las semillas, lavándoles otra vez para posteriormente dejarles en tachos plásticos a orear, un grupo de semillas amarillas y blancas por $48 \mathrm{~h}$ y el otro grupo por $72 \mathrm{~h}$. Luego de este proceso se procedió a sembrar las semillas de menor tiempo de oreo, ubicando por bandeja $0.45 \mathrm{~kg}$ de semillas, al siguiente día se sembraron las semillas restantes.

Los primeros cuatro días solo se utilizó agua, regando dos veces al día, a las 8 am y a las 5 pm. Los fertilizantes se aplicaron al quinto y al octavo día, la micorriza se aplicó en una dosis de $2 \mathrm{~g} / \mathrm{L}$ de agua, y raízfares, con una dosis de $2 \mathrm{~mL} / \mathrm{L}$ de agua. Posteriormente solo se utilizó agua, hasta la cosecha que se realizó a los 12 días.

Los instrumentos de precisión que se emplearon fueron una balanza con capacidad de $10 \mathrm{~kg}$, una cinta métrica, y una bomba de fumigar de $6 \mathrm{~L}$.

Variables de respuesta. Se evaluaron las variables peso y altura de plantas, tamaño de raíz, estas variables se definen por su importancia productiva en forraje hidropónico. La variable raíz, se consideró por los tipos de enraizadores empleados.

Análisis estadísticos. Los datos de cada una de las variables de respuesta que satisficieron o aproximaron a los supuestos de distribución normal y homogeneidad de varianzas, se analizaron de acuerdo al modelo estadísticos planteado (Gabriel et al. 2017).

Sobre la base del modelo mencionado, se realizaron análisis de varianza para probar hipótesis acerca de los efectos fijos y comparaciones de medias mediante la prueba de rango múltiple de tukey al p $<0.05$ de probabilidad. El análisis de varianza también sirvió para estimar los componentes de varianza para los efectos aleatorios. Los análisis indicados se realizaron utilizando el paquete estadístico infostat (Gabriel et al. 2017).

\section{Resultados}

Los resultados determinaron diferencia estadística significativa a nivel de variedad, con un valor de $\mathrm{p}=0.04$, definiéndose al maíz blanco como estadísticamente diferente del maíz amarillo, de igual mane- 
ra se determinó que el fertilizante con un valor de $\mathrm{p}=0.0012$ tuvo una interacción altamente significativa, siendo el enraizador inorgánico el de mejores resultados, de igual manera se establece interacción significativa entre los factores tiempo-fertilizante con un valor de $\mathrm{p}=0.03$. El mejor resultado lo presento el maíz blanco con enraizador orgánico con 48 horas de oreo, y peso de $1.60 \mathrm{~kg}$ en bandejas de 40 x $50 \mathrm{~cm}$, empleando $0.45 \mathrm{~kg}$ de maíz por bandeja (Tabla 1).

Tabla 1 resumen de la producción de FVH de Maíz amarillo, blanco, con dos tiempos de oreo y con dos tipos de enraizadores

\begin{tabular}{|c|c|c|c|c|c|c|}
\hline Variedad & Tiempo de oreo & Tipo de enraizadores & Tratamientos & Peso K & Altura & Tamaño de Raíz \\
\hline \multirow{4}{*}{ Maíz Amarillo } & \multirow{2}{*}{48 horas } & Inorgánico & V1O1E1 & $1.57^{\mathrm{ab}}$ & 12.58 & $10.20^{\mathrm{ab}}$ \\
\hline & & Orgánico & V1O1E2 & $1.28^{\mathrm{b}}$ & 14.03 & $9.07^{\mathrm{b}}$ \\
\hline & \multirow[t]{2}{*}{72 horas } & Inorgánico & V2O1E1 & $1.36^{\mathrm{ab}}$ & 15.64 & $9.86^{\mathrm{ab}}$ \\
\hline & & Orgánico & V2O1E2 & $1.29^{\mathrm{b}}$ & 16.18 & $7.91^{\mathrm{b}}$ \\
\hline \multirow{4}{*}{ Maíz Blanco } & \multirow{2}{*}{48 horas } & Inorgánico & VIO2E1 & $1.60^{\mathrm{a}}$ & 13.24 & $10.78^{\mathrm{a}}$ \\
\hline & & Orgánico & V1O2E2 & $1.34^{\mathrm{ab}}$ & 13.50 & $9.09^{\mathrm{ab}}$ \\
\hline & \multirow[t]{2}{*}{72 horas } & Inorgánico & V2O2E1 & $1.50^{\mathrm{ab}}$ & 14.94 & $12.49^{\mathrm{a}}$ \\
\hline & & Orgánico & V2O2E2 & $1.47^{\mathrm{ab}}$ & 15.37 & $9.65^{\mathrm{ab}}$ \\
\hline
\end{tabular}

Los literales en las columnas corresponden a la prueba de significación según Tukey al 0.05

Con respecto a la altura no se estableció efectos estadísticamente diferentes entre variedades y fertilizantes, sin embargo se destaca que el factor tiempo, con un valor de $\mathrm{p}=0.0023$, establece que este si influyo estadísticamente a nivel de altura, determinándose que con $72 \mathrm{~h}$ de oreo, se obtuvo mejores resultados. Se indica además que no existió interacción entre los otros factores (Tabla 1).

Con relación al tamaño de raíz, hubo mejores resultados del tratamiento con maíz blanco y de enraizador inorgánico con $12.49 \mathrm{~cm}$ y $10.72 \mathrm{~cm}$, y tiempos de oreo de 72 y $48 \mathrm{~h}$ respectivamente. Hubo diferencias significativas entre variedades $(\mathrm{p}=0.0242)$, siendo la variedad maíz blanco la mejor. Hubo asimismo diferencias altamente significativas $(\mathrm{p}=0.0008)$ entre el enraizador inorgánco y orgánico, donde el enraizador inorgánico tuvo mejores resultados (Tabla 1). En lo referente al tiempo y a las interacciones variedad-tiempo, variedadfertilizantes, tiempo-fertilizantes, y variedadtiempo-fertilizante, no hubo diferencias estadísticas significativas.

\section{Discusión}

Los beneficios de la agricultura han sido significativos. En los últimos 50 años a producción de granos para la alimentación del hombre y de animales domésticos se ha duplicado, principalmente por el incremento en los rendimientos de los cultivos debido a mayores aplicaciones de fertilizantes, agua y pesticidas, mejoramiento genético y otras tecnologías que surgieron de la Revolución Verde (Tilman et al. 2002). Sin embargo, en las prácticas agrícolas que han incrementado visiblemente el suplemento mundial de alimentos, que ocasionan indudablemente impactos negativos sobre el medioambiente, obligando a buscar metodologías agrícolas más sustentables. En numerosos países, como por ejemplo Australia, la industria lechera es uno de los principales usuarios del agua de riego (Linehan et al. 2004) y se encuentra presionada para utilizarla con mayor eficiencia (Armstrong 2004). Asimismo, es comúnmente reportado que para producir $1 \mathrm{~kg}$ de carne se requiere entre 3 y $12 \mathrm{~kg}$ de alimento obtenido a partir de especies forrajeras que requieren 
grandes cantidades de agroquímicos y agua de riego para lograr altos rendimientos.

Utilizando la metodología de producción de FVH se puede cosechar anualmente 15-25 t de materia seca (López-Aguilar et al. 2009). Este rendimiento es equivalente al de la alfalfa, sorgo o maíz, pero en una superficie 100 veces menor y sin utilización de agroquímicos. Para obtener de 1 a 8 kg de MS de alimento para el ganado cultivando especies mediante métodos convencionales se emplea $1 \mathrm{~m}^{3}$ de agua de riego, mientras que utilizando este mismo volumen de agua en la producción de FVH se obtienen alrededor de $100 \mathrm{~kg}$ de MS de forraje de buena calidad nutricional para alimentar diversos tipos de ganado (FAO 2001). Aunque se han evaluado métodos de riego recientemente incorporados a los sistemas agrícolas, como el riego por goteo subterráneo, para producir pasturas con menores cantidades de agua (Godoy-Ávila et al. 2003), la realidad es que el UEA aún sigue manteniendo valores bajos de 1.6-1.9 $\mathrm{kg}$ MS $\cdot \mathrm{m}^{-3}$ en comparación con los calculados para el sistema de producción de FVH. En nuestro estudio aun cuando no se llega a este nivel de detalle, sugiere que se hagan estos estudios, para obtener resultados acordes a nuestra realidad.

Por otra parte en varias investigaciones realizadas (Lopez- Aguilar et al. 1999) se demostró que la calidad nutricional del FVH es valiosa, ya que se le han encontrado características apropiadas, como excelente gusto y un alto contenido de proteína cruda (Müller et al. 2005). Estos aspectos no fueron tratados en este estudio, lo que sugiere que todavía existen varios temas de investigación que deben desarrollarse.

Es importante resaltar que el FVH es un alimento con el suficiente valor nutricional para considerarlo como un suplemento alimenticio ideal para elevar la condición nutricional del ganado, principalmente en 149 zonas áridas y semiáridas donde es común que los animales pasen por periodos de subnutrición en diferentes etapas de su vida (Espinoza et al. 2007). Otro criterio comúnmente utilizado para determinar la calidad del forraje es la digestibilidad. En las paredes celulares de los vegetales existe una fracción digerible y otra no digerible. El contenido de FDA es una cuantificación de la fracción indigerible. En el FVH la FDA varía con el tiempo de cosecha, observándose valores menores en la etapa inicial y valores mayores en la etapa final. Este comportamiento es similar a otras plantas forrajeras en las cuales se ha reportado que conforme la planta madura, su contenido de FDA aumenta, y la ingestión y digestibilidad se reduce (Van Soest et al. 1978).

Los resultados obtenidos implican una producción de maíz blanco de $3.53 \mathrm{lb}$, por lb de semilla sembrada, y en el caso del maíz amarillo fue en el mejor de los casos de 3.46, producción relativamente baja frente a Vargas-Rodríguez (2008) que en su investigación obtuvo una producción 1: 4.3 de biomasa, en este sentido González et al. (2015), en su investigación de FVH de maíz blanco y amarillo obtuvo una producción promedio de 1: 4.7 entendiendo que la explotación de estos niveles es posible, siempre y cuando se maneje de manera adecuada las fluctuaciones de temperatura, la cual por estar en el trópico, en horas pico puede superar los $35^{\circ} \mathrm{C}$, y los cultivos hidropónicos deben estar en un promedio de $25^{\circ} \mathrm{C}$, Otro aspecto importante es el uso de semilla con un alto poder germinativo.

En lo que respecta a la altura, se obtuvo un promedio de $14 \mathrm{~cm}$, muy por debajo de MoralesRodríguez (2012), investigador que obtuvo un promedios de $25 \mathrm{~cm}$ en el mismo tiempo de cosecha, Meza-Carranco (2005) en su tesis investigativa obtuvo resultados que fluctuaron entre los 20 a $35 \mathrm{~cm}$ 
de altura, en las variedades de maíz Pioneer 3025 y

Pioneer 31 G98 respectivamente.

El tamaño de raíz en el experimento realizado se encuentra entre 10 a $12 \mathrm{~cm}$, menor al realizado por Meza-Carranco (2005) que en su investigación realizada probando dos variedades de maíz, obtuvo en la variedad Pioneer $302514 \mathrm{~cm}$, y de la variedad Pioneer $31 \mathrm{G} 98$ en promedio $22.5 \mathrm{~cm}$, destacando que las condiciones climáticas fluctuaron entre los $19^{\circ}\left(\right.$ a $\left.25^{\circ} \mathrm{C}\right)$ temperatura ideal para la producción de FVH.

\section{Conflictos de intereses}

Esta investigación fue realizada con el financiamiento de la Universidad Estatal del Sur de Manabí y no presenta conflictos de interés.

\section{Agradecimientos}

Los autores expresan sus agradecimientos a la Universidad Estatal del Sur de Manabí, por el financiamiento de la presente investigación.

\section{Literatura citada}

Abarca-Reyes P, Aguirre-Aguilera C, Silva-Rubio L, Mora-López D, Carrasco-Jimenéz J. Producción de forraje verde hidropónico para la pequeña agricultura. Boletin INIA 2014; 321: 1-26.

Armstrong DP. Water use efficiency and profitability on an irrigated dairy farm in northern Victoria: a case study. Aust J Exp Agric 2004; 44: 137-44.

Ecuale. Provincia Manabí. Disponible en http://www.ecuale.com/manabi/. (2016).

Eluniverso. Manabí dividida entre la sequía y las inundaciones. Disponible en http://www.eluni verso.com/noticias/2015/03/02/nota/4611686/

manabi-dividida-sequia-inundaciones.

Espinoza-Villavicencio JL; Palacios-Espinosa A, Ávila-Serrano N, Guillén-Trujillo Ariel, de Luna-de la Peña R, Ortega-Pérez R, et al. La ganadería orgánica, una alternativa de desarrollo pecuario para algunas regiones de México: una revisión. Interciencia 2007;32(6):385-90.

FAO. El Manual Técnico "Producción de Forraje Verde Hidropónico” . En TCP/ECU/066 (A) Mejoramiento de la disponibilidad de alimen tos en los Centros de Desarrollo Infantil del INNFA. Santiago de Chile: Oficina Regional de la FAO para América Latina y el Caribe. 2001.

Gabriel J, Castro C, Valverde A, Indacochea B. Diseños experimentales: Teoría y práctica para experimentos agropecuarios. Grupo COMPAS, Universidad Estatal del Sur de Manabí (UNE SUM), Jipijapa, Ecuador; 2017. p. 146.

Godoy-Ávila C, Pérez-Gutiérrez A, Torres-E CA, Hermosillo LJ, Reyes JI. Uso de agua, produc ción de forraje y relaciones hídricas en alfalfa con riego por goteo superficial. Agrociencia 2003;37(2):107-15.

González E, Ceballos J, Benavidez O. Producción de forraje verde hidropónico de maiz Zea mays. L en invernadero con diferentes niveles de silicio. Rev Cienc Agr 2015;32(1):75-83.

Linehan CJ, Armstrong DP, Doyle PT, Johnson F. A survey of water use efficiency on irrigated dairy farms in northern Victoria. Austr J Exp Agric 2004;44:131-6.

López-Aguilar R, Murillo-Amador B, RodríguezQuezada G. El Forraje Verde Hidropónico (FVH) una alternativa de producción de alimento para el ganado en zonas áridas. Interciencia 2009;34(2):121-6. 
Maldonado-Torres R, Álvarez-Sánchez ME, Acevedo DC, Ríos-Sánchez E. Nutrición mineral de forraje verde hidropónico. Rev Chapingo SerHortic 2013;19(2):211-23.

Meza-Carranco Z. Evaluación de densidades de maíz y densidad de siembra en la producción de forraje verde hidropónico. [Tesis de Licenciatura]. Universidad Autónoma de Nuevo León; 2005. p. 86.

Morales-Rodríguez HJ, Gómez-Danés AA, JuárezLópez P, Loya-Olguín L, Ley de Coss A. Forraje verde hidropónico de maíz amarillo (Zea maíz L.) con diferente concentración de solución nutritiva. Abanico Vet 2012;2(3): 20-8.

Müller L, Manfron P, Santos O, Medeiros S, Haut V, Dourado D, et al. Producción y composición bromatológica de forraje hidropónico de maíz (Zea mays L.) con diferentes densidades de siembra y días de cosecha. Brasil. Zootecnia Tropical 2005;23(2):105-19.

Rivera R, Moronta M, González-Estopiñán E, González D, Perdomo D, García DE, et al. Producción de forraje verde hidropónico de maíz en condiciones de iluminación deficiente. Zootecnia Trop 2010;28(1):33-41.
Tilman D, Cassman KG, Matson PA, Naylor R, Polasky S. Agricultural sustainability and intensive production practices. Nature 2002; 418(6898):671-7.

Van Soest PJ, Mertens DR, Deinum B. Preharvest factors influencing quality of conserved forage. J Anim Sci 1978;47(3):712-20.

Vargas-Rodríguez CF. Comparación productiva de forraje verde hidropónico de maíz, arroz y sorgo negro forrajero. Agron Mesoam 2008;19(2): 233-40.

Villavicencio PA. Producción de Forraje Hidropónico. Boletín INIA No 285. Instituto de Investigaciones Agropecuarias, Centro Regional de Investigación La Platina, Santiago, Chile; 2014. p. 44. 